\title{
Management of the LHCb Readout Network
}

\author{
Guoming Liu and Niko Neufeld
}

\begin{abstract}
The LHCb event building relies on a readout network based on Gigabit Ethernet (GbE). The readout network is extremely performance critical, sophisticated monitoring at all levels is essential for the successful operation of the data acquisition for the experiment. LHCb uses a commercial supervisory control and data acquisition (SCADA) system called PVSS for its experiment control system (ECS), which consists of varied sub-systems. For the efficient operation of the experiment, it is crucial that all parts of the system are monitored and controlled through the same interface in a coherent way. The network monitoring is implemented in the same framework. We show here how a large scale network can be monitored using tools originally made for industrial supervisory control. The status of the network devices and their ports, their connectivity and the traffic are monitored in this system. Alarms can be issued to inform the experiment operators and online network experts about errors such as dropped packets or broadcast-storms. Reports and long-term monitoring using powerful trending tools are possible.
\end{abstract}

Index Terms-DAQ, network management, readout network.

\section{INTRODUCTION}

$\mathbf{L}$ $\mathrm{HCb}[1]$ is an experiment dedicated to the study of the decays of B-hadrons produced at the large hadron collider (LHC). The collision is at a centre of mass energy up to $14 \mathrm{TeV}$ at a rate of $40 \mathrm{MHz}$, and the events that can be detected by $\mathrm{LHCb}$ are produced at about $10 \mathrm{MHz}$. This data rate is reduced by a two-level trigger system. The first level trigger (L0) is constructed in dedicated hardware, which reduces the rate to accepted events to $1 \mathrm{MHz}$ based on the transverse momentum information measured in the calorimeter and muon sub-systems. At this rate the entire detector is read out, since the full event data are required for LHCb physics. Simulation studies indicate that at the nominal conditions expected at the LHC, the total event-size should be about $35 \mathrm{kB}$, so the readout network throughput is about $35 \mathrm{~GB} / \mathrm{s}$. The second level or high level trigger (HLT) is purely implemented in software running on a large farm of commodity servers, which further reduce the output rate to about $2 \mathrm{kHz}$. The trigger and data acquisition (DAQ) rely directly on a dedicated network (denoted as readout network or DAQ network), which is based on Gigabit Ethernet and consists of a core switch and more than 50 edge switches.

In the following sections, the architecture of the readout network, the management of the readout network including monitoring and configuring, will be described.

Manuscript received May 20, 2009; revised August 12, 2009. Current version published April 14, 2010.

G. Liu is with Ferrara University, Ferrara I-44100, Italy, and CERN, Geneve 23, CH-1211, Switzerland (e-mail: gliu@ cern.ch).

N. Neufeld is with the PH Department, CERN, Geneve 23, CH-1211, Switzerland.

Digital Object Identifier 10.1109/TNS.2009.2035694

\section{ARChitecture OF DAQ AND THE NETWORK}

The whole LHCb data readout system [2] is shown in Fig. 1. The core network consists of a single chassis switch E1200i, which is provided by Force10 Networks [3]. This is one of the highest density Gigabit Ethernet (GbE) switch available to date, it supports up to 14 line-cards of $90 \mathrm{GbE}$ ports, namely $1260 \mathrm{GbE}$ ports in total. The E1200i has a $3.5 \mathrm{Terabit} / \mathrm{sec}$ (Tbps) switch fabric capacity, and 2 billion packet/sec (Bpps) full-mesh forwarding capacity, which matches the requirement of the LHCb readout network. In the commissioning phase and the first year of data-taking, the data rate to read out is expected to be below $20 \%$ of the full nominal rate. To take full advantage of Moore's law in computing capability, the full deployment of the data readout system will be done as late as possible. Currently only two line-cards were deployed in the core switch, and an aggregation layer was introduced to meet the requirement of LHCb commissioning. The aggregation layer is used to connect the links from each readout board, while deliberately overcommiting its uplinks into the core network. As shown in Fig. 2, four or five readout links in the HP Procurve 5412 switches [4] are converged to one uplink connected to the core switch E1200i.

The LHCb data acquisition system works in a push mode. The event data are sent from the front end electronics (FEE) of the detectors via approximately 5000 optical or analog links. These links are fed into about 330 readout boards called TELL1 [5], where the data are pre-processed and zero-suppressed. The readout boards reduce the data rate by a factor of 4 to 8 depending on the attached sub-detector. The readout boards are connected to the readout network with up to four Gigabit Ethernet ports. The real number of links connected to the network depends on the data rate of each readout board.

A custom network transport protocol was defined for the data transfer from the readout boards to farm-nodes, which is called multi-eventfragment protocol [6]. This is a light-weight, unreliable datagram protocol over IP like user datagram protocol (UDP), there is no retransmission mechanism. Multi-Eventfragment Protocol allows to assemble the data from multiple events into a multi-event packet (MEP) in order to reduce the overhead from protocol headers and to mitigate the packet rate at the receiving nodes. In Multi-Eventfragment Protocol, the packing factor, namely the number of events in a single MEP, can be tuned to gain a better efficiency. The destination IP address and packing factor are assigned by the timing and fast control (TFC) system, which is used for all synchronous information transfer in the experiment. Since there is no back-pressure in the protocol for the data readout, an external throttle mechanism is used to avoid overflow of buffers at all levels. For readout boards, throttle signal can be fed back to the readout supervisor, the central module of the 


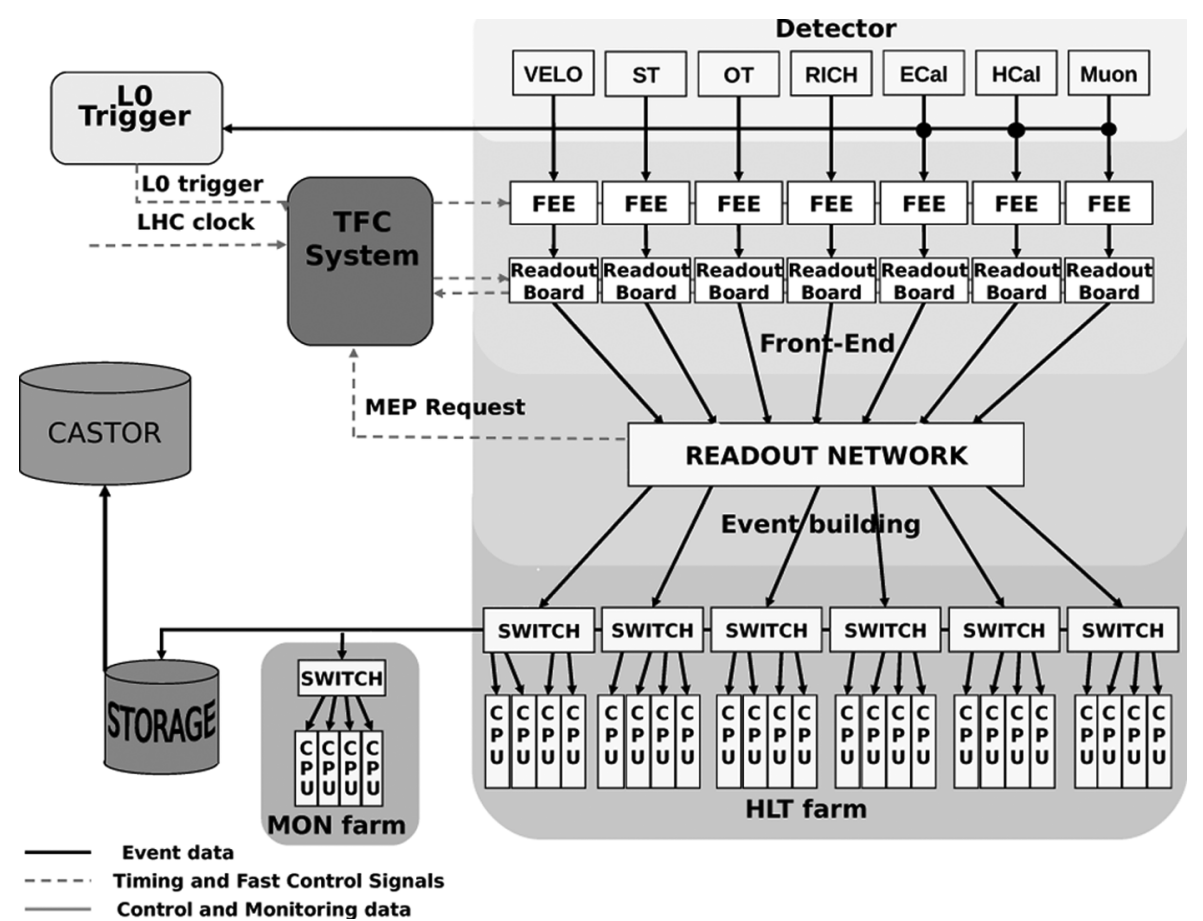

Fig. 1. Architecture of $\mathrm{LHCb}$ data readout system.

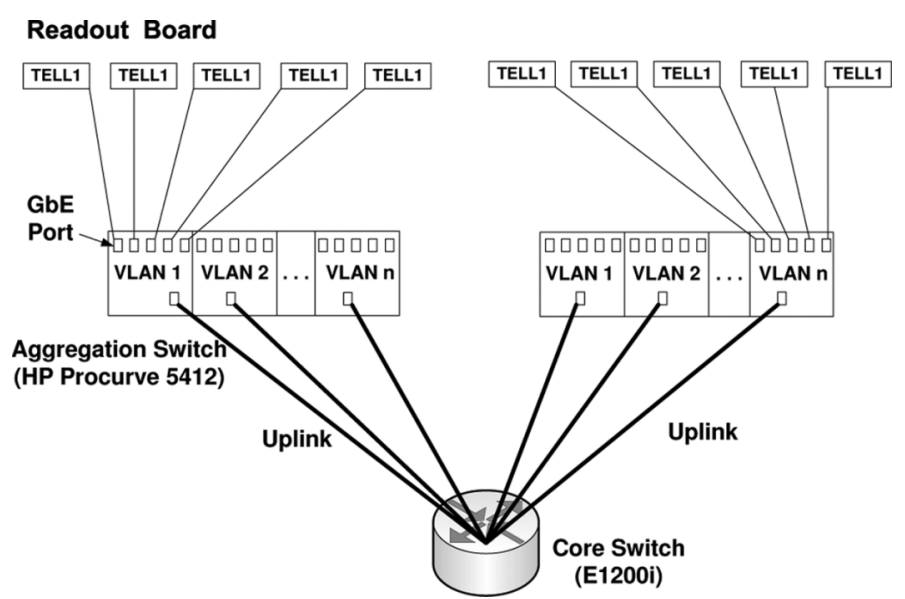

Fig. 2. Diagram of aggregation layer.

TFC system. The farm nodes announce their availability by sending a MEP request to the readout supervisor.

In the core switch E1200i, the event data are routed to the edge switches, then the edge switches further forward the data to the HLT CPU farm. For the data readout, the edge switches are operated at Layer 2. The CPU farm is physically installed in 50 racks, where a single edge switch HP Procurve 3500 is installed in each rack and connected to the core switch E1200i with up to 12 uplinks. In each edge switch, currently only two uplinks are used for the commissioning. Link aggregation (LAG) [7] is used for the uplinks. LAG uses multiple network links in parallel to increase the link speed beyond the limits of any single link, and to increase the redundancy for higher availability. With the same hardware, LAG provides high bandwidth logical link and redundancy without extra cost. In the edge switch, a virtual local area network (VLAN) must be created for each uplink to the core switch to avoid network loops. Since the maximum port member of a LAG is eight in the edge switch, the 12 uplinks are divided into two LAGs, which reduces the VLAN number from 12 to 2 , and makes the configuration and management of the edge switches easier.

The HLT software running in the farm node further reduces the event rate to about $2 \mathrm{kHz}$. Accepted events are sent off the farm nodes, routed by the edge switch to the $\mathrm{LHCb}$ online storage cluster, where all the selected events are written into raw data files. The size of the raw data file is normally 2 GB. The raw data files are registered in a run-database and transferred to the CERN mass-storage facility CASTOR as soon as they are closed. For security reasons, a firewall is deployed in the $\mathrm{LHCb}$ online network. Only those trusted nodes in CERN computing center are allowed to access the LHCb online storage cluster.

\section{Network MANAGEMENT: Monitoring}

The LHCb online system consists of three separated networks, the control network, the data network, and the management network. The dedicated data network is divided into two networks logically, the readout network and the storage network. The readout network will be used exclusively for the data readout, transferring the event data from the readout boards to the HLT farm nodes. The readout network is a large network and extremely performance critical, so sophisticated monitoring at all levels is essential for the successful operation of the data acquisition for the experiment.

\section{A. Architecture of the Network Monitoring System}

LHCb uses supervisory control and data acquisition (SCADA) [8] system PVSS [9] and Joint COntrols Project (JCOP) [10] framework for its experiment control system. PVSS is a commercial software system from Austrian 


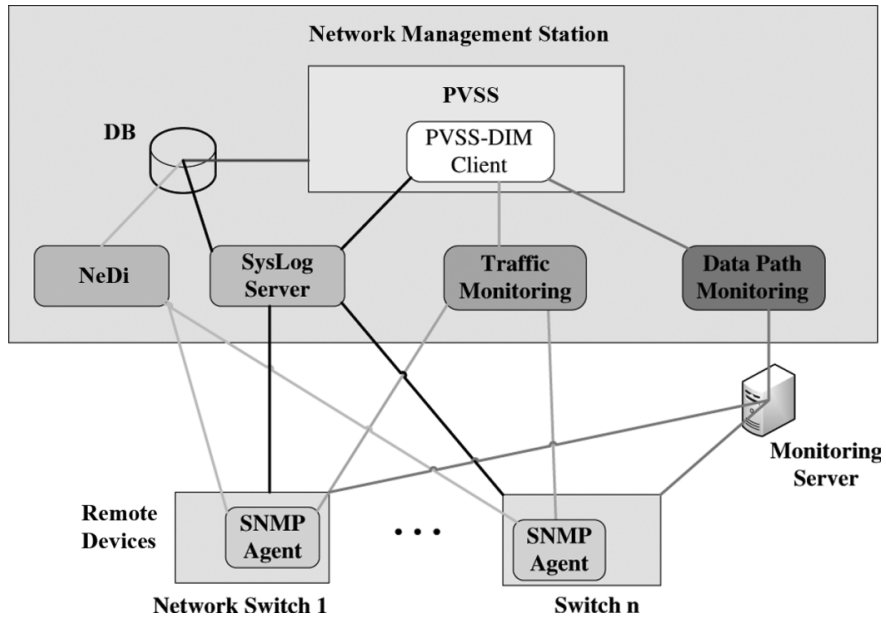

Fig. 3. Architecture of the network monitoring system.

company ETM, and has been used extensively in industry for the supervision and control of industrial processes. PVSS can be used to connect hardware (or software) devices, acquire the data produced by the devices and use the data for their supervision and control, i.e., to monitor their behavior and to initialize, configure and operate them. JCOP is a common project setup between the four LHC experiments to define a common architecture and a framework to be used for their control systems. The experiment control system (ECS) of LHCb handles the configuration, monitoring and operation of all experimental equipment involved in the different activities of the experiment. For the efficient running of the experiment by non-expert operators, it is crucial that all parts of the system are monitored and controlled through the same interface in a coherent way, so the central ECS of LHCb provides a framework to all the sub-systems for their configuration and control. The network monitoring system is implemented in this framework as a coherent part. According to the LHCb ECS guideline [11], the finite state machines (FSM) for the network monitoring is implemented with the tool provided by the JCOP Framework. The FSM describes the states and possible transitions in network domains, and provides a common look and feel as described in [11].

The architecture of the monitoring system is shown in Fig. 3. The supervision layer of the monitoring system is implemented with the SCADA system PVSS. In the network monitoring system, PVSS is used to monitor the status of the network devices and the throughput of the switch interfaces. The system is mostly based on simple network management protocol (SNMP) [12], which is supported by all the network devices of LHCb. SNMP is an application layer protocol that facilitates the exchange of management information between managed network devices and the management station. SNMP enables network administrators to monitor the network throughput, manage network performance, configure network devices, find and solve network problems. SNMP driver is provided in PVSS, but the performance is quite low, it is not suitable for a large scale system. To improve efficiency, the other front-end processes are used to query data from the network devices via SNMP. As shown in Fig. 3, the open source

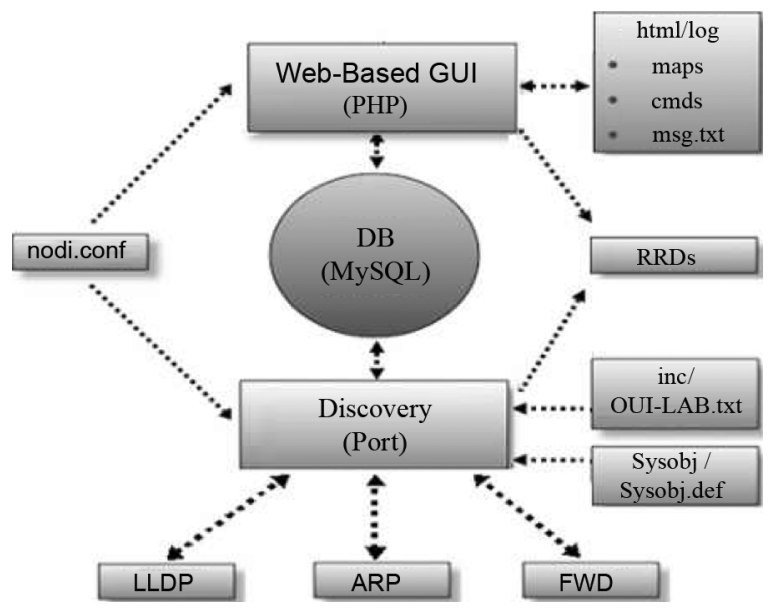

Fig. 4. Architecture of NeDi.

network discovery tool NeDi is used to explore the network topology, discover network devices (switches, routers) and the attached nodes (the hosts and the readout boards); the SysLog Server is used to collect the syslog from the network devices, $\log$ all the messages and send those alarm messages to PVSS; a front-end process for the traffic monitoring is written to collect the switch status and the interface traffic information; the data path monitoring is used to monitor the routing status for all the data flow. The details are described in the following sections.

\section{B. NeDi: Network Topology Discovery}

$\mathrm{NeDi}[13]$ is an open source system for network discovery, management, and inventory, of which the architecture is shown in Fig. 4. NeDi has been originally developed for Cisco devices and limited non-Cisco devices, now is transplanted to the $\mathrm{LHCb}$ online system for the network topology discovery even though the LHCb network devices are not from Cisco. The network discovery is based on link layer discovery protocol (LLDP) [14], which is supported by all the network devices in LHCb online system. LLDP is a vendor-neutral Layer 2 protocol that allows a network device to advertise its identity and capabilities on the local network, and to learn about each other.

NeDi queries the LLDP MIB (Management Information Base) from the network devices via SNMP. With a seed device as a starting point, NeDi begins to query for the LLDP neighbors, and then the neighbors of those neighbors, and so on until all the devices have been discovered in the network. Based on the LLDP information, the map of the network topology can be learned accurately. To discover the nodes in the network, NeDi queries the address resolution protocol (ARP) entries and the MAC address for which the switch bridge has the forwarding information. Then it parses all the switch tables it knows about for node information. In this way, the nodes can be discovered and inventoried, including the IP address, MAC address, NIC type, and the switch port to which it is currently attached.

All the topology information are stored in the database, which is used by PVSS.

\section{SysLog Server}

A syslog server is setup to listen on port 514 for receiving the syslog messages from the network devices. Syslog allows a 
machine to send the event notification messages across IP networks to the event message collector. The syslog packets are sent in clear text, and have three distinct parts: priority, header and message. The priority part represents both the facility and severity of the message. A facility in syslog is a class of messages, the facility LOG_NEWS is assigned to the network devices in the $\mathrm{LHCb}$ online system.

When some network devices run into problems, error messages will be generated and sent to the syslog server as configured in the network device. Once the syslog messages are received, the syslog server will log all the messages in a text file. For those messages with higher priority above warning, the syslog server stores the message in the database, and sends it to PVSS via distributed information management (DIM) [15] for further processing. DIM is a communication system for distributed/mixed environments, it provides a network transparent inter-process communication layer, and it is widely used in $\mathrm{LHCb}$ experiment control system. With the syslog server, it is possible to monitor the status of the hardware, detect hardware problems, e.g., high temperature, fan failure. This helps isolate the network device problem, and allows the network administrators to take some proactive actions before a real network device problem.

\section{Data Path Monitoring}

From the network's point of view, there are three stages for the LHCb DAQ: event data from the readout boards to the HLT farm, selected event data from the HLT farm to the LHCb online storage, raw data files from the $\mathrm{LHCb}$ online storage to CERN CASTOR. In all these stages, the data transmissions are working at Layer 3, and the traffics are routed through varied paths. In the operation of 2008, some edge switches stopped routing traffic after a powercut due to a bug in the switch operating system. So it is critical to monitor the routing in each stage for the DAQ. A simple tool to monitor reachability is ping, which sends Internet control message protocol (ICMP) echo request packets to the target host and listens for ICMP echo response replies. The status of data paths can be known by analyzing the statistical summary of the echo response packets received in the hosts.

In the readout boards, full features of ICMP are not implemented, ping can not be launched for the test. To monitor the data path from the readout boards to the HLT farm, a monitoring server is used to simulate the readout board, and it is connected to the same VLAN as the readout boards in the core switch. The monitoring is divided into two parts: the path from the readout boards to the monitoring server and the path from the monitoring server to the HLT farm.

1. The monitoring server detects the status of the readout boards using a lower level of protocol ARP. A script is implemented to check the availability of the readout boards using raw socket. In this script, the monitoring server sends the ARP requests for the destination IPs in parallel through multiple threads. The readout board will respond to the request for itself with its own MAC address. The status of the interfaces of the readout boards can be found out by analyzing the received ARP responses.

2. The monitoring server is used to simulate the readout board and scan the availability of the HLT nodes with ping since both ends support fully ICMP. A multi-threaded program is launched from monitoring server to ping all the HLT nodes, the result is obtained for each node by analyzing the ICMP replies received.

Combining the result of these two steps, the status of the data path from the readout boards to the HLT farm can be learned.

For monitoring the routing from the HLT nodes to the $\mathrm{LHCb}$ online storage, the method is similar. The monitoring server has an interface connected to the storage subnet, and the same program is launched to scan the availability of the HLT nodes for the storage nodes. For the monitoring of the routing from the $\mathrm{LHCb}$ storage to CERN CASTOR, only the destinations are different. For this scanning, the destinations are a set of nodes, which are allowed to access the LHCb online storage by the firewall. The list of trusted nodes are fetched dynamically from the CERN LANDB, which is a network database used for the management of CERN campus network.

At the end of the scanning for each stage, the result is summarized and published to PVSS via DIM. In the DIM service, the status of each nodes or readout boards are published for every stage. The DIM clients of the PVSS network monitoring system subscribe to these DIM services, and will receive the updates for each scan.

\section{E. Traffic Monitoring}

A light-weight front-end process called SNMP collector is written to query data from the network devices via SNMP. The SNMP collector logically consists of three primary modules: SNMP message transport module, SNMP message processing module, and DIM message exchange module.

The SNMP message processing module deals with encoding and decoding of SNMP messages, and the SNMP message transport module is used for exchanging SNMP messages over network based on Berkeley sockets. Both the SNMP message transport module and the SNMP message processing module are implemented based on the Net-SNMP [16] library. In the managed network devices, the properties of the managed object are collected in the MIB, which is accessible through object identifier (OID). To collect the required information, the SNMP collector sends out a SNMP request message with the request type and the corresponding object identifier, and then waits for the response from the managed device. When SNMP messages are received, they are decoded based on the request object identifier, request type and the data type.

Because Net-SNMP library is not thread-safe, an independent process is running to query the information from each single switch. All the SNMP collectors are launched from the same network management station to collect the information from the switches in LHCb data readout network. To improve the efficiency, the process uses GET-BULK operation in SNMP version $2 \mathrm{C}$ to query the values in bulk instead of one by one.

The process queries the uptime of the device, the interface information (e.g., ifName, ifDescr, ifSpeed), the interface counters (ifInOctets, ifInPackets, ifOutOctets, ifOutPackets), and the interface errors (ifInError, ifOutDiscard). The interface counters are used to calculate the port bandwidth utilization along with the device uptime. The interface errors indicate the error of each port in the switch. There are two vendors for the network devices in LHCb online system, Force10 and HP Procurve. The HP Procurve switches update SNMP counters almost in real time, 
but the Force10 switches update SNMP counters every 15 seconds. To get an accurate traffic measurement, the query interval must be a multiple of the interval which the switches update the counters, and the query should be in the safe time slot which is not close to the time switches update the counters. Currently the query interval is set to 60 seconds for all the devices.

The SNMP collector also acts as a DIM server, which exchanges the information with the upper layer PVSS. PVSS communicates with DIM server via PVSS-DIM which provides a bridge between PVSS datapoints (either full structures or single items) and DIM services/clients. In PVSS, the device data are structured as a datapoint (DP) of a pre-defined datapoint type (DPT). A DPT describes the data structure of the device and a DP contains the information related to a particular instance of such a device. The DPT structure is user definable and can be as complex as one requires and may also be hierarchical. In the network monitoring system, PVSS acts as a DIM client which subscribes to the service provided by the DIM server. Datapoints are created for each switch to receive the raw data sent by DIM servers. A callback function is called to parse the raw data when the datapoint is updated. The raw data will be separated for each interface and the values will be set to the datapoints for the interfaces. The status of the switch is set according to the traffic information. Alarms are enabled in each switch and each interface. If some errors occur, e.g., the packet drop rate is above the threshold, or the non-unicast packet rate is too high, an alarm will be triggered, and all the error information will be logged. Based on the trending tool of the JCOP framework, a panel is developed to display the histograms of the current input bandwidth utilization, output utilization, and the errors for each ports, which allows to see the overview of all ports in a switch. Another panel is developed to display the traffic trending (including port utilization, packet rate, error) of the interface. All the traffic information is archived in an Oracle database. The system can generate the report for a given period by query the archived data. This can be used to analyze the traffic model and optimize the network system.

\section{F. Finite State Machine of the Network Monitoring System}

On the top layer of the network monitoring system, the interface is a finite state machine based on the LHCb ECS framework. The FSM of the network monitoring is hierarchical and built as a tree of interconnected components. At the higher levels, the control nodes called control units (CU), allow an operator to take the control of the associated sub tree of the system, and at the very lower level, the control nodes called devices units (DU) connect to the real hardware components that they supervise. As shown in Fig. 5, the state of the switch is monitored, including the hardware status and the traffic throughput status. The uplinks between these switches, and the routing status of the data path, are monitored as well.

According to the guide for the FSM design [11] and the property of the readout network, three states (Ready, Error and Off) are defined to indicate the state of the readout network. The state changes propagate along the tree from the hardware component that changes its status, up to the control node CUs. Currently this FSM is for monitoring only, no interactive behavior is implemented, but this is feasible and will be improved.

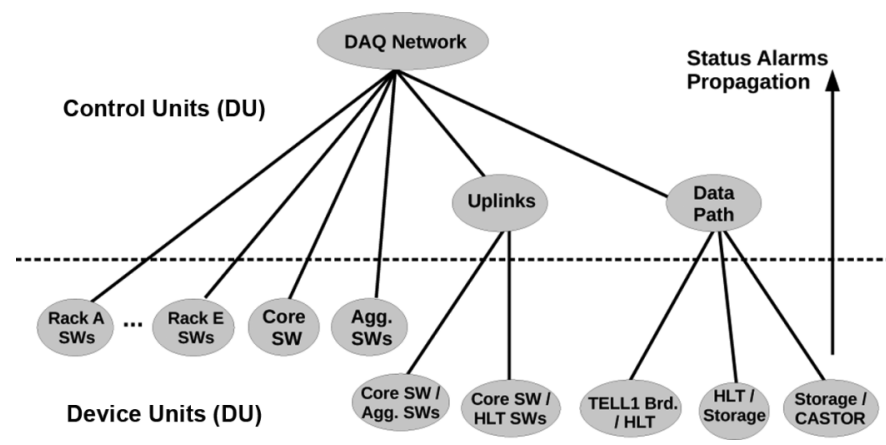

Fig. 5. Architecture of the FSM for the Network Monitoring System.

\section{Network MANAGEMENT: CONFIGURATION}

The LHCb online network system is quite large, where several kinds of switches are deployed. The configuration of the different devices with different command sets is burdensome to the network administrator. There are several commercial solutions for network configuration management (NCM), offering an extensive set of features. Since the LHCb online network is quite static after the full system is installed, only a few features are essential for configuring network devices.

Currently a set of custom Python scripts is used for configuring the network devices.

1. A script is used to initialize the switch before its deployment. In this script, the common configuration for all the switches is applied. The common configuration includes user and password, network time protocol (NTP), SNMP, syslog receiver, etc.

2. A script is written to manage the configuration file, which can be used to backup the configuration file to a tftp server, or to roll back to the previous configuration.

3. A script is written to upgrade the firmware for the switches. Most of the devices support booting from two memory locations (primary and secondary). The script will copy the last validated firmware version to the redundant flash, then upgrade the firmware in the active flash. This makes sure there is at least one valid firmware in the switch.

Beside, the command line interface (CLI) access to remote network devices is implemented in NeDi to some extent. It is based on the perl module Perl::SSH::Perl, which implements an SSH (Secure Shell) client. It is compatible with both the SSH-1 and SSH-2 protocols. Net::SSH::Perl enables to execute commands on remote machines simply and securely. On the web-based GUI, a batch of commands can be committed in a set of switches which can be defined. With the CLI access and the web GUI, it is easy to do some common configuration, e.g., the firmware upgrade, VLAN configuring, etc.

\section{Network Diagnostics}

Several tools have been developed to troubleshoot and understand the problems that can occur in the readout network.

\section{A. High Speed Traffic Monitoring}

The monitoring described above is mainly for analyzing the performance of the global LHCb network system. The query interval is too long to troubleshoot some problems. A script is implemented to monitor a limited set of interfaces at high speed, 
and thus the bandwidth utilization plots can be produced with a fine time resolution. For the HP Procurve switch, the script queries the counter using SNMP. Because of the long update of the SNMP counter in Force10 switch, the counters are queried using the CLI, which updates the counter very fast. In order not to saturate the CPU resource, the traffic is measured at $1 \mathrm{~Hz}$ by default.

\section{B. Sflow Sampler}

SFlow [17] is a standard for monitoring computer networks, based on statistical sampling. It is applicable to high speed networks (at gigabit speed or higher). The sFlow monitoring system consists of an sFlow Agent (embedded in the switch/router) and an sFlow collector. The sFlow Agent resides anywhere within the path of the packet, and combines the sampled packet header, ingress and egress interfaces, sampling parameters, and interface counters into sFlow datagrams and forwards them to the sFlow collector. A simple collector and analysis package has been developed to analyze the traffic characteristics. It is possible to identify network conversations, i.e., the source and destination IP or MAC addresses. This is often used to troubleshoot packet losses. For example, the packets sent from the readout board are not received by the HLT node, this is usually caused by incorrect destination MAC address or IP address. With the sFlow tool, it is easy to find out this kind of problem.

\section{CONCLUSION}

The network monitoring system is developed with PVSS and the JCOP framework. The system can work in an efficient way with the custom front-end processes to query the SNMP MIB from the network devices. The FSM describes the states in network domains, and provides a common look and feel for the operators. The readout network is monitored at varied levels: hardware status, bandwidth utilization, error information and routing status etc. Alarms can be issued to inform the experiment operators and network administrators in case of errors.

The system is running on the network management station to monitor the status of the readout network, but not yet fully integrated into the central ECS of LHCb. The SNMP polling interval is 60 seconds, which is limited by the SNMP counter update interval of the Force10 switches. With the interval of 60 seconds, both the SNMP collector and the PVSS supervision system work stably. In the past several months, the system not only monitored the network status, but also contributed to isolate DAQ problems.

However, there are still some improvements to be done.

1. There are too many archived data and alerts defined in the PVSS project, it takes a long time to read the previous value when the project restarts. Some unnecessary or unimportant archiving of data and alert should be removed.

2. Some interactive behaviors should be implemented in the FSM for the experiment operators. The behaviors include some basic operations on the switches, e.g., reset.

3. The auto-generated daily report and long-term analysis report should be implemented.

\section{ACKNOWLEDGMENT}

The authors would like to thank the CERN IT department, in particular the teams of IT/CS (campus networking) and IT/CO (controls) for their tight collaboration and effective support.

\section{REFERENCES}

[1] LHCb Collaboration, "LHCb reoptimized detector design and performanc," Tech. Design Rep., 2003 [Online]. Available: http://lhcb-tp. web.cern.ch/lhcb-tp/postscript/tp.ps

[2] LHCb Collaboration, "LHCb online," Tech. Design Rep., 2001 [Online]. Available: http://lhcb-comp.web.cern.ch/lhcbcomp/General/Publications/onlinetdr.pdf

[3] “Force10 E-Series Manuals,” Force10 Networks, San Jose, CA, 2009.

[4] "Management and Configuration Guide for the ProCurve Series 3500yl, 6200yl, 5400zl, and 8200zl Switches," HP ProCurve Networking, CA, 2009.

[5] G. Haefeli et al., "The LHCb DAQ interface board TELL1," Nucl. Instrum. Methods Phys. Res. A, vol. 560, pp. 494-502, 2006.

[6] B. Jost and N. Neufeld, "Raw-data transport format," Tech. Doc., 2004 [Online]. Available: https://edms.cern.ch/file/499933/1/transportformat-v5.0.pdf

[7] Link Aggregation, IEEE Std. 802.3ad, 2000.

[8] S. A. Boyer, SCADA: Supervisory Control and Data Acquisition, 3rd ed. North Carolina: Int. Soc. of Automation (ISA), 2004.

[9] PVSS: Process Visualization and Control System. ETM Professional Control. [Online]. Available: http://www.pvss.com/

[10] LHC Experiments Joint Control Project. [Online]. Available: http:// itco.web.cern.ch/itco/Projects-Services/JCOP/

[11] P. Duval, "Guide for ECS FSM Design in LHCb Detectors," Tech. Doc. [Online]. Available: https://edms.cern.ch/file/655828/3/ LHCb_ECS_FSM_Guidelines.pdf

[12] W. Stallings, SNMP, SNMPv2, SNMPv3, and RMON 1 and 2, 3rd ed Reading, MA: Addison-Wesley Professional, 1999.

[13] NeDi: Network Discovery. [Online]. Available: www.nedi.ch

[14] Link Layer Discovery Protocol, IEEE Std. 802.3ab, 2005.

[15] DIM: Distributed Information Management. [Online]. Available: http://dim.web.cern.ch/dim

[16] Net-SNMP. [Online]. Available: http://www.net-snmp.org/

[17] P. Phaal, S. Panchen, and N. McKee, "InMon corporation's sFlow: A Method for monitoring traffic in switched and routed networks," RFC 3176, 2001. 